\title{
Reaping Wnt after calming Hippo: Wnt and Hippo signaling cross paths in lung cancer
}

\author{
Thomas Andl ${ }^{1}$, Yuhang Zhang $^{2}$ \\ ${ }^{1}$ Burnett School of Biological Sciences, University of Central Florida, Orlando, FL, USA; ${ }^{2}$ Division of Pharmaceutical Sciences, College of Pharmacy, \\ University of Cincinnati, Cincinnati, OH, USA \\ Correspondence to: Dr. Thomas Andl. Burnett School of Biomedical Sciences, University of Central Florida, 12722 Research Parkway, Orlando, FL \\ 32826, USA. Email: thomas.andl@ucf.edu. \\ Provenance: This is an invited Editorial commissioned by Section Editor Dr. Chunlin Ou (Cancer Research Institute of Central South University, \\ Changsha, China). \\ Comment on: Han Q, Lin X, Zhang X, et al. WWC3 regulates the Wnt and Hippo pathways via Dishevelled proteins and large tumour suppressor 1, \\ to suppress lung cancer invasion and metastasis. J Pathol 2017;242:435-47.
}

Submitted Sep 14, 2017. Accepted for publication Oct 03, 2017.

doi: $10.21037 /$ jtd.2017.10.29

View this article at: http://dx.doi.org/10.21037/jtd.2017.10.29

It has become clear in recent years that many signaling pathways influence each other and work cooperatively to regulate biological processes, such as the generation of complex multicellular tissues, organs, and organisms. These complex interactions are the subject of systems biology, a young and still poorly established approach in defining the complexities of life and the intricate interactions of major signaling pathways (1). Before systems biology can truly thrive, sometimes simple steps must be taken to determine the crosstalks between individual signaling pathways. A recent paper published by Han et al. (2) in the fournal of Pathology illuminated just such a step. This piece of the puzzle will aid in our understanding of how complex organisms form during embryogenesis, how they are maintained in adult life, and how they are prevented from falling prey to the chaotic forces of cancer. Han et al. have delved deep into the interactions of the two major pathways that have been shown to control most biological processes in animals. These two pathways are Wnt and Hippo signaling. Han et al. identified WWC 3 as a new player that connects both pathways. They have also been shown how aberrant regulation of WWC3 can contribute to lung tumorigenesis. WWC3 is like other members of the WWC family: a scaffolding protein that can maintain Hippo signaling and suppress YAP1 activity, e.g., by activating LATS kinases.

Like so many other cancer-associated genes and pathways, Wnts, Wnt, and Hippo signaling were originally identified as key regulators of embryonic development in the fruit fly Drosophila $(3,4)$. Wnts are secreted proteins that signal through a family of receptors called Frizzleds. These receptors initiate a cascade of events that lead to the inhibition of the APC-AXIN-GSK3B complex, one of the major negative regulators of cytoplasmic and nuclear beta-catenin protein pools (Figure 1). Accumulation of nuclear beta-catenin and transcriptional activation of target genes by binding to LEF1/TCF or other transcription factors/regulators-such as the Hippo target YAP1-are the key events in canonical Wnt signaling, generally resulting in pro-tumorigenic effects in cancer cells. It has become clear that Wnts control many processes during metazoan embryogenesis, functioning as morphogens that influence cellular responses dependent on a concentration gradient reminiscent of other developmentally important regulators (6). However, it also became clear that Wnt signaling is relevant for processes in adult tissues, as well as during tumorigenesis (7). Due to its importance for the evolution of the metazoan body plan, the regulation of Wnt signaling has evolved into a complex system that includes feedback and feedforward loops and well-established interactions with the Hippo pathway and other pathways $(1,8)$.

There are striking similarities between the architecture of the canonical Wnt pathway and Hippo signaling. Both pathways center on an inhibitory complex in the cytoplasm 


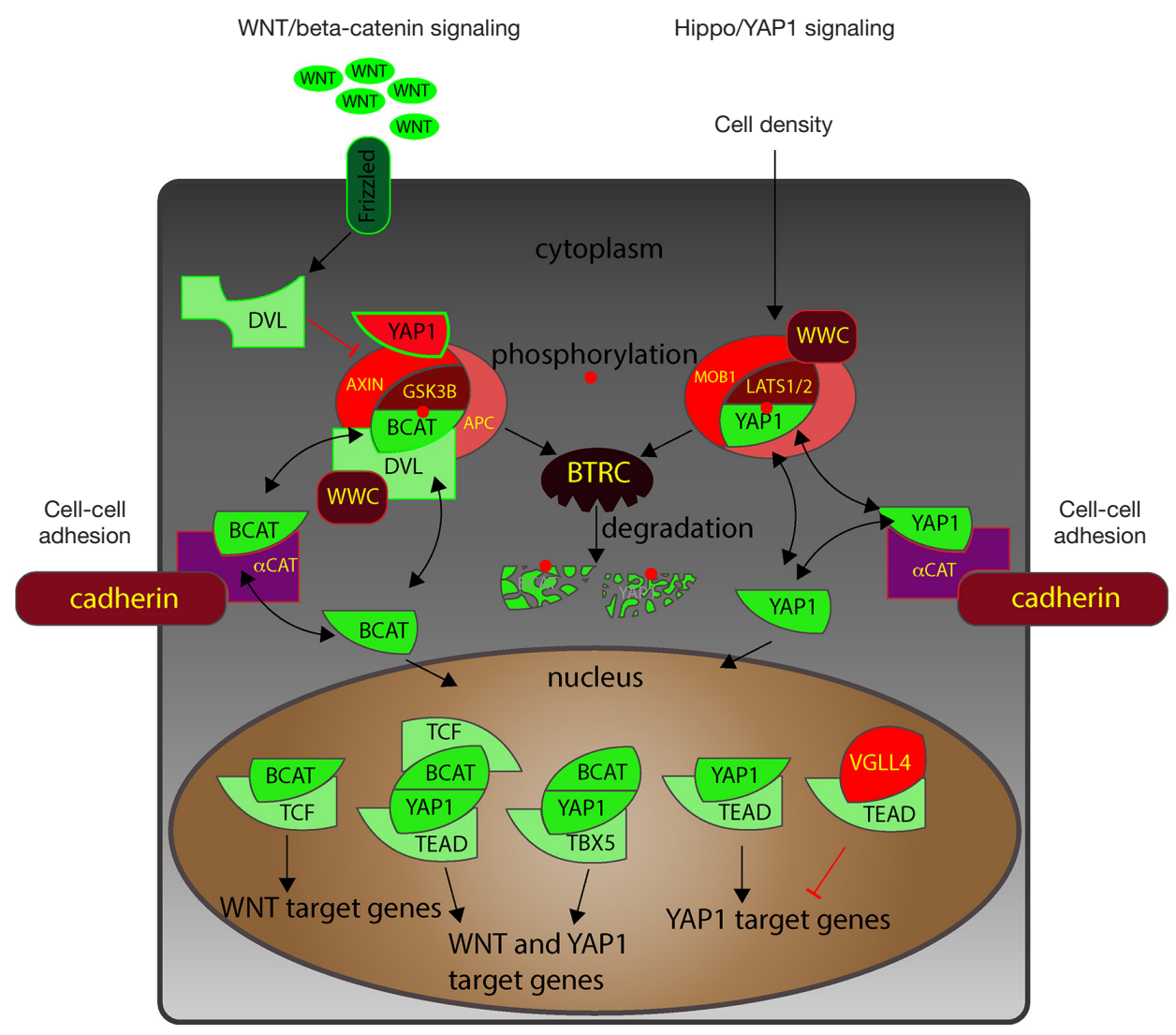

Figure 1 Model of Wnt and Hippo signaling network that either promotes or inhibits Wnt signaling through cytoplasmic and nuclear interactions of the two pathways. Major regulators of Hippo signaling are cell-cell adhesion via cadherin-based adherens junctions [e.g., through alpha-catenin $(\alpha C A T)$ ] and via tight junctions to integrate cell density. Canonical Wnt signaling is initiated through binding of Wnt ligands to Frizzled receptors. In both Wnt/beta-catenin (BCAT) and Hippo/YAP1 pathways, phosphorylation (red circles) at specific sites by GSK3B and LATS1/2, respectively, target BCAT and YAP1 for BTRC-mediated degradation. Several signaling components are utilized to mediate Wnt-Hippo signaling coordination: e.g., BCAT, YAP1, WWC3 (WWC), DVL, and AXIN interact with components of the other signaling pathway. Another shared component is the destruction pathway BTRC (also known as E3-ubiquitin ligase $\beta$-TrCP). YAP/ WWTR1 can be essential components of the BCAT destruction complex by interacting with AXIN (5). Thereby, Wnt signaling also can regulate Hippo/YAP1 signaling since YAP1 is sequestered through AXIN in this destruction complex and is released upon WNT signaling activation. Both BCAT degradation and YAP1 degradation can be mediated through BTRC. In the nucleus several complexes exist that contain components of both pathways suggesting cooperation of Wnt and Hippo signaling upon Wnt activation.

(APC-AXIN-GSK3B and LATS1/2-STK3/4-SAV1) that prevents the key factors of the pathway (beta-catenin and YAP1/WWTR1*) from entering the nucleus where YAP1 binds to transcription factors of the TEAD family and where $\beta$-catenin binds to LEF1/TCF transcription factors (Figure 1). Both pathways are also intricately linked to the outside environment of the cell through adherens junctions and tight junctions. These links to the cell surface allow both pathways - as in the case of Hippo signaling and to some extent in the case of Wnt signaling-to integrate information about the environment into the activity of the signaling pathway, e.g., by accounting for cell density or

\footnotetext{
* Here we use standard NCBI gene names rather than colloquial names, e.g., the official name for the downstream target of Hippo signaling is not TAZ but WWTR1. TAZ should not be used since this name is reserved for another unrelated gene, Tafazzin (human Gene ID: 6901). The same is true for STK3 and 4 which we prefer over MTS1 and 2.
} 
orientation of the cell in the tissue organization.

Beta-catenin has been identified as a key component of a adherens junctions, which connect the extracellular matrix and membrane surface to the actin cytoskeleton. Similarly, YAP1 is associated with membrane proteins that mediate cell-cell adhesion [mainly tight junctions, e.g., through AMOT, but also adherens junctions through alphacatenin (9)]. It is tempting to speculate that both pathways, Wnt and Hippo, may cooperate to regulate the activity and the phenotype of cells by integrating the cellular context into life decisions, such as proliferation, differentiation, or senescence. Beyond this "cell density" aspect both pathways may also coordinate in managing the immune phenotype and apoptosis of cancer cells $(10,11)$. Han et al. (2) have expanded our knowledge and have shown that an upstream component of Hippo signaling, WWC3, not only inhibits YAP1, it also inhibits beta-catenin accumulation in the nucleus.

Beyond its "traditional" role in Hippo signaling of binding to LATS proteins, WWC3 can bind to DVLs (regulators of the AXIN-APC-GSK3 complex), thereby maintaining the inhibitory activity of the complex (Figure 1). This finding by Han et al. further strengthens the notion of a tight coordination between Hippo and Wnt signaling, as YAP1/WWTR1 have also been shown to target DVLs (12). Adding WWC3 into this network may enhance the cooperation between the two pathways. However, this causes WWC 3 or WWC family members to become weak spots in the anti-tumor defense system. Han et al. showed that WWC3 expression is down-regulated during lung cancer progression and its expression may become a novel marker for poor prognosis of non-small cell lung cancer (2). WWCs are highly similar and they all have the ability to regulate LATS1/2 protein function. It is unclear whether all WWCs can interfere with Wnt signaling or whether WWC3 is the major WWC family member that coordinates Wnt and Hippo function. Some datasets suggest that $\mathrm{WWC} 1$ is expressed at higher levels in normal lungs than WWC3 and is suppressed at a greater rate than WWC3 (NCBI GEO dataset GDS1312).

Unfortunately, many details remain to be worked out before WWC3, Wnt, and Hippo signaling can be used in clinical diagnosis. It therefore remains to be seen whether these genes and pathways can ever be integrated into therapeutic approaches (13). The issue of redundancy between WWC family members is also unresolved.

Findings on LATS1/2 may tell a cautionary tale of opposing effects of these pathways in cancer cells and the cancer-supporting microenvironment. Although the WWC binding partners LATS1/2 are generally regarded as tumor-suppressor genes in the Hippo pathway, their complete absence in cancer cells can be a disadvantage. The loss of LATS1/2 can induce an anti-dsRNA interferon response and activate the immune system to attack these LATS1/2-deficient tumor cells (14). Therefore, restoring certain functions of the Hippo pathway in cancer cells may actually promote their ability to evade the immune system. Cancer cells, however, rarely lose their LATS1/2 expression completely. Indeed, they may actually recruit microRNAs, which have been shown to target LATS1 and LATS2 (5) to reduce their expression to growth-beneficial and YAP1inducing levels. Despite this reduction, they maintain enough LATS1/2 to avoid the negative consequences of their loss, e.g., activation of the immune system (14) or catastrophic events during mitosis (15). LATS1/2 levels can thereby be sufficiently reduced and YAP1 can be activated. As such, one could also make the cautious argument that these pathways are currently poor direct targets for cancer therapy. The expression levels and activity of certain components may have to be in a certain range to achieve clinically successful therapies. This could prove difficult to accomplish in patients.

Another important factor to consider in the interplay between Wnt and Hippo signaling is the fact that cytoplasmic ("inactive") YAP1/WWTR1 can inhibit DVLs and prevent Wnt signaling $(11,16)$. Barry et al. (16) have shown that elevated levels of cytoplasmic YAP1 can inhibit intestinal crypt proliferation. In fact, reduced levels of YAP1 actually enhanced proliferation in crypts and may promote aggressive tumor growth. This may explain why so few real human tumors exhibit high levels of YAP1 and why YAP1 is often low in the nuclei of human cancer cells. On the other hand, this may be due to other mechanisms of YAP1 activity since there seems to be no "correlation between YAP1 nuclear localization or expression and YAP1 dependency" in cancer cell lines (11).

Furthermore, there is mounting evidence that YAP1/ WWTR1 can interact with beta-catenin containing protein complexes and regulates either the stability of beta-catenin in the cytoplasm or the interaction of unphosphorylated YAP1 with beta-catenin in the nucleus to coordinate gene expression $(11,17)$. Complexes of YAP1/TEAD and betacatenin/TCF have been detected and a subset of Wnt/ beta-catenin target genes may rely on nuclear YAP1/ TEAD complexes for full induction in response to Wnt ligands (18). These data suggest that in addition to the 
complex interactions between Wnt and Hippo signaling components in the cytoplasm, both pathways can cooperate in the nucleus to execute gene expression programs that affect proliferation, organ size, tissue repair, and tumor growth. These experiments confirm the elegant findings of Rosenbluh et al. (11) that have shown that in cancer cell lines that depend on nuclear beta-catenin signaling, YAP1 plays a crucial role in mediating beta-catenin gene expression regulation. The drawback of the Rosenbluh et al. study is the explanation that TBX5 mediates the transcriptional response of the beta-catenin/YAP1 complex in beta-catenin-dependent cell lines: RNAseq and cDNA microarray analyses (e.g., cBioPortal, GTEx portal, Human Protein Atlas RNAseq data) do not seem to support significant expression of TBX5, especially in vitro but also in vivo in the vast majority of cell lines and tissues, and therefore it is hard to understand how a non-expressed gene can mediate beta-catenin-driven transcriptional responses.

However, such experiments elucidate the complex interactions between several major signaling pathways that form byzantine signaling networks. This complexity and interactivity further highlights that a systems biology approach is warranted to understand gene expression regulation (1). For example, Wnt, Hippo, and Notch signaling have been studied in the context of liver tumorigenesis and it has been shown that these three pathways work together in a tissue- and context-dependent way (19). Kim et al. have shown that upon inactivation of Hippo signaling in mouse liver, Notch signaling is activated. The two pathways are connected via a positive feedback loop that results in the loss of YAP1/WWTR1 activation upon Notch signaling inactivation. As expected, in mouse liver tumors initiated by loss of Hippo signaling, Wnt/ beta-catenin signaling is induced. Surprisingly, loss of betacatenin expression in this model system leads to accelerated tumorigenesis. This paradoxical finding seems to depend on beta-catenin's role as a Wnt signaling mediator and not its function in cell adhesion, since loss of beta-catenin in adherens junctions can be compensated for by gammacatenin, which is not functional in Wnt signaling.

These data highlight how interactions between pathways can result in, at first glance, paradoxical findings: e.g., the signaling of pro-tumorigenic pathways Wnt and YAP1 can have opposing effects on tumor growth (18). Furthermore, in some experimental systems a majority of beta-catenin regulated genes are dependent on WWTR1 (TAZ) (20). Although this reliance on Wnt target genes appears to be dependent on cytoplasmic interactions between beta- catenin and WWTR1 (TAZ), co-regulation of a subset of Wnt target genes can also be achieved by the cooperation of beta-catenin/TCF and YAP1/WWTR1/TEAD transcriptional complexes (18). On the other hand, Park et al. observed in their experiments that activation of noncanonical Wnt signaling-i.e., Wnt signaling independent of beta-catenin-resulted in increased levels of WWTR1 and YAP1, increased expression of YAP1 target genes, increased TEAD-dependent migration and differentiation, and expression of Wnt signaling inhibitors, all of which leads to reduced canonical Wnt signaling (21). This shows that Hippo and Wnt signaling can influence each other in many perplexing ways; they can either cooperate or YAP1/WWTR1 can inhibit canonical Wnt signaling. This multilayer, cytoplasmic, and nuclear network of interactions just between Wnt and Hippo signaling suggests that cells and tissues have a versatile toolbox at their disposal to regulate cell behavior in a context-dependent fashion. This is supported by the often contradictory results in mouse models on the role of Wnt and Hippo signaling on tumorigenesis and tissue repair, especially when both pathways are altered. Further studies are needed to define how exactly these interactions play out in lung development and lung cancer and whether there are subgroups of lung cancers with simultaneous Hippo inhibition and canonical Wnt signaling activation or if these alterations are mutually exclusive, as may be the case in liver cancer (19).

It is fascinating to note that these major signaling pathways and molecules used in embryogenesis as morphogens induce strict, well-defined, and reproducible patterns under normal physiological conditions. These molecules become abused and ill-regulated in cancer cells, thus producing chaotic and destructive tissues. Lung cancer is no exception to this subversion of developmental programs, such as Wnt and Hippo signaling. However, exploiting this knowledge and restoring the functionality of these pathways is a daunting task. The immense progress in tumor immunology and immune therapy has shown the power of targeting nontumor cells rather than tumor cells (22).

A recent paper by Chung et al. investigated non-tumor cells in breast cancer at the single cell transcriptome level and identified that the majority of them are immune cells with immune-suppressive signatures (23). This emerging theme of targeting the tumor-associated microenvironmentincluding immune cells, fibroblasts, and macrophages (23) will be the next frontier in cancer research. Since the tumor microenvironment and tissue is an aberrant and often wound-like version (24) of the normal tissue, the pathways 
that guide tissue maintenance and repair (e.g., Wnt, Hippo, and TGFbeta) will still play a central role in understanding tumorigenesis and tumor progression.

And despite the constant, confusing and scary emergence of new details on how these pathways are networked [e.g., the link of Hippo and Wnt pathways through the methyltransferase SETD7 (25)], these new details may also open new opportunities to target these pathways in order to diminish cancer cell growth and survival.

The studies by Han et al. and other groups on the interaction of Wnt and Hippo pathways provide a foundation on which such novel therapies may be developed. Many additional questions must be resolved and more detailed maps of cancer signaling networks must be drawn in order to predict when a Wnt storm can arise after Hippo (2) has been calmed, or whether calming a Hippo can also cause major storms without Wnt signaling activation [e.g., (19)].

\section{Acknowledgements}

None.

\section{Footnote}

Conflicts of Interest: The authors have no conflicts of interest to declare.

\section{References}

1. Attisano L, Wrana JL. Signal integration in TGF- $\beta$, WNT, and Hippo pathways. F1000Prime Rep 2013;5:17.

2. Han Q, Lin X, Zhang X, et al. WWC3 regulates the Wnt and Hippo pathways via Dishevelled proteins and large tumour suppressor 1, to suppress lung cancer invasion and metastasis. J Pathol 2017;242:435-47.

3. Baker NE. Molecular cloning of sequences from wingless, a segment polarity gene in Drosophila: the spatial distribution of a transcript in embryos. EMBO J 1987;6:1765-73.

4. Pan D. The hippo signaling pathway in development and cancer. Dev Cell 2010;19:491-505.

5. Luan L, Shi J, Yu Z, et al. The major miR-31 target genes STK40 and LATS2 and their implications in the regulation of keratinocyte growth and hair differentiation. Exp Dermatol 2017;26:497-504.

6. Lander $\mathrm{AD}$. Morpheus unbound: reimagining the morphogen gradient. Cell 2007;128:245-56.
7. Holland JD, Klaus A, Garratt AN, et al. Wnt signaling in stem and cancer stem cells. Curr Opin Cell Biol 2013;25:254-64.

8. Konsavage WM Jr, Yochum GS. Intersection of Hippo/ YAP and $W n t / \beta$-catenin signaling pathways. Acta Biochim Biophys Sin (Shanghai) 2013;45:71-9.

9. Schlegelmilch K, Mohseni M, Kirak O, et al. Yap1 acts downstream of $\alpha$-catenin to control epidermal proliferation. Cell 2011;144:782-95.

10. Hugo W, Shi H, Sun L, et al. Non-genomic and Immune Evolution of Melanoma Acquiring MAPKi Resistance. Cell 2015;162:1271-85.

11. Rosenbluh J, Nijhawan D, Cox AG, et al. $\beta$-Catenin-driven cancers require a YAP1 transcriptional complex for survival and tumorigenesis. Cell 2012;151:1457-73.

12. Varelas X, Miller BW, Sopko R, et al. The Hippo pathway regulates $\mathrm{Wnt} /$ beta-catenin signaling. Dev Cell 2010;18:579-91.

13. Andl T, Zhou L, Yang K, et al. YAP and WWTR1: New targets for skin cancer treatment. Cancer Lett 2017;396:30-41.

14. Moroishi T, Hayashi T, Pan WW, et al. The Hippo Pathway Kinases LATS1/2 Suppress Cancer Immunity. Cell 2016;167:1525-39.e17.

15. Yang $\mathrm{X}$, Yu K, Hao Y, et al. LATS1 tumour suppressor affects cytokinesis by inhibiting LIMK1. Nat Cell Biol 2004;6:609-17.

16. Barry ER, Morikawa T, Butler BL, et al. Restriction of intestinal stem cell expansion and the regenerative response by YAP. Nature 2013;493:106-10.

17. Heallen T, Zhang M, Wang J, et al. Hippo pathway inhibits Wnt signaling to restrain cardiomyocyte proliferation and heart size. Science 2011;332:458-61.

18. Jiao S, Li C, Hao Q, et al. VGLL4 targets a TCF4TEAD4 complex to coregulate Wnt and Hippo signalling in colorectal cancer. Nat Commun 2017;8:14058.

19. Kim W, Khan SK, Gvozdenovic-Jeremic J, et al. Hippo signaling interactions with $\mathrm{Wnt} / \beta$-catenin and Notch signaling repress liver tumorigenesis. J Clin Invest 2017;127:137-52.

20. Azzolin L, Panciera T, Soligo S, et al. YAP/TAZ incorporation in the $\beta$-catenin destruction complex orchestrates the Wnt response. Cell 2014;158:157-70.

21. Park HW, Kim YC, Yu B, et al. Alternative Wnt Signaling Activates YAP/TAZ. Cell 2015;162:780-94.

22. Zhou L, Yang K, Andl T, et al. Perspective of Targeting Cancer-Associated Fibroblasts in Melanoma. J Cancer 2015;6:717-26. 
23. Chung W, Eum HH, Lee HO, et al. Single-cell RNA-seq enables comprehensive tumour and immune cell profiling in primary breast cancer. Nat Commun 2017;8:15081.

24. Dvorak HF. Tumors: wounds that do not heal-redux. Cancer Immunol Res 2015;3:1-11.

Cite this article as: Andl T, Zhang Y. Reaping Wnt after calming Hippo: Wnt and Hippo signaling cross paths in lung cancer. J Thorac Dis 2017;9(11):4174-4179. doi: 10.21037/ jtd.2017.10.29
25. Oudhoff MJ, Braam MJS, Freeman SA, et al. SETD7 Controls Intestinal Regeneration and Tumorigenesis by Regulating Wnt/ $\beta$-Catenin and Hippo/YAP Signaling. Dev Cell 2016;37:47-57. 